\title{
A Case of Evans Syndrome and Unstable Angina
}

\author{
Saad Bin Jamil ${ }^{\text {a, d }}$, Iqra Patolia, Mehrunissa Kazim ${ }^{\mathrm{b}}$, \\ Syed Hassan Abbas ${ }^{\text {a }}$, Zain Alic
}

\begin{abstract}
Evans syndrome (ES) is characterized by autoimmune hemolytic anemia (AIHA) and immune-mediated thrombocytopenia. It is more common in the pediatric population than in adults. ES has been reported to be associated with thrombotic events and rarely can lead to acute coronary syndrome (ACS). There have been only a few reported cases of ACS secondary to ES. We present an interesting case of ES with unstable angina (UA) which had a limited response to oral and intravenous (IV) steroids requiring rituximab. A 64-year-old male with past medical history significant for hypertension, hyperlipidemia, diabetes mellitus and coronary artery disease, presented to the emergency room complaining of a 2 -week history of chest pain, shortness of breath and hematuria. Physical examination indicated splenomegaly but was otherwise unremarkable with no petechiae or rash. Labs showed hemoglobin of $9.6 \mathrm{~g} / \mathrm{dL}$, platelet count $58 \times 10^{3} / \mu \mathrm{L}$, troponin $<0.03 \mathrm{ng} / \mathrm{mL}$, lactic acid $2.5 \mathrm{mmol} / \mathrm{L}$ and with parameters indicative of hemolysis, evidenced by elevated lactate dehydrogenase, low haptoglobin and elevated bilirubin levels. Electrocardiography (EKG) demonstrated ST depression in leads I, aVL, V5 - V6 and T wave inversions in lead III and aVL, which were new compared to previous EKG. Peripheral blood smear indicated spherocytes. Direct antiglobulin test was positive for immunoglobulin $\mathrm{G}(\operatorname{IgG})$. Patient was admitted for ES and initially treated with oral prednisone $80 \mathrm{mg}$ daily. He was also diagnosed with UA thought to be possibly secondary to ES. He then underwent cardiac stress test which showed mild reversible inferior apical ischemia. Cardiac catheterization revealed $95 \%$ stenosis of proximal left circumflex artery requiring single drug eluding stent placement and dual antiplatelet therapy. Patient continued to have anemia despite blood transfusions, although platelet count improved. Prednisone was transitioned to high-dose IV dexamethasone, and patient was also started on rituximab which resulted in stabilization of anemia. The presentation of ES with ACS is a rare occurrence. ACS can be challenging to manage as stent placement may be required followed by dual antiplatelet
\end{abstract}

Manuscript submitted August 21, 2021, accepted September 11, 2021

Published online September 29, 2021

aDepartment of Internal Medicine, St. Mary's Hospital, Waterbury, CT 06706, USA

bepartment of Internal Medicine, Holyoke Health Center, Holyoke, MA 01040, USA

'Abington Memorial Hospital, Philadelphia, PA 19001, USA

${ }^{d}$ Corresponding Author: Department of Internal Medicine, St. Mary's Hospital, Waterbury, CT 06706, USA. Email: saadbinjamil@hotmail.com

doi: https://doi.org/10.14740/jmc3777 therapy. Treatment of ES involves steroids followed by rituximab, IV immunoglobulin (IVIG) or splenectomy for non-responsive cases. Early intervention and management can prevent mortality and morbidity.

Keywords: Evans syndrome; Unstable angina; Autoimmune hemolysis; Thrombosis; Thrombocytopenia; Intravenous immunoglobulin; Rituximab

\section{Introduction}

Evans syndrome (ES) presents with autoimmune hemolytic anemia (AIHA) [1]. In addition, patients can also present with neutropenia and immune thrombocytopenic purpura (ITP). Neutropenia, AIHA and ITP can occur at the same time or present sequentially [2]. ES is more prevalent in the pediatric population as compared to adults [1].

ES can be categorized as primary or secondary which is dependent on presence or absence of associated connective tissue diseases/autoimmune diseases [1]. Primary type is considered to be idiopathic whereas secondary ES is associated with other diseases or syndromes such as systemic lupus erythematosus, common variable immunodeficiency and non-Hodgkin lymphoma [3]. When associated with other diseases, the management (and outcomes) may be different as opposed to the management of primary ES, which makes correct diagnosis of integral importance [3].

ES is associated warm AIHA, in which immunoglobulin $\mathrm{G}$ (IgG) antibodies react with red blood cell (RBC) surface antigens at body temperature, as opposed to cold AIHA [4].

The presenting signs and symptoms of ES can be variable. Although primarily a chronic condition, it is characterized by relapsing remitting flares which can be challenging to manage. It is rare for patients to develop thrombotic events. Although venous thromboembolic events (VTEs) have been reported in some cases, there are no clearly established incidence rates of arterial thrombosis in patient with ES. According to one retrospective study evaluating 68 patients of ES, six patients developed cardiovascular events over a period of 4.8 years among which four had acute coronary syndrome (ACS) [3]. The study also reflected that risk of AIHA-related complication such as thrombosis may supersede the risk of thrombocytopenia-related complications such as bleeding [3]. Non-ST segment elevation myocardial infarction presenting with ES is extremely rare [5]. Treatment involves steroids, although patients who do not 
respond to steroids, may require more aggressive interventions such as administration of rituximab or intravenous immunoglobulin (IVIG). In some cases, with significant thrombocytopenia, splenectomy may also be considered although the benefits of splenectomy are not well established. We present an interesting case of ES with unstable angina (UA) requiring rituximab and intravenous (IV) steroids.

\section{Case Report}

\section{Investigations}

A 64-year-old male with past medical history significant for hypertension, hyperlipidemia, diabetes mellitus and coronary artery disease with two prior stent placements in right coronary artery and patent ductus arteriosus, presented to the emergency room (ER) due to chest pain and shortness of breath for 2 weeks. He also endorsed fatigue and gross hematuria. Patient did not have any petechiae, bleeding elsewhere or rash. The review of systems was otherwise unremarkable.

\section{Diagnosis}

Physical examination demonstrated splenomegaly and palmar pallor. Otherwise, examination was unremarkable. Electrocardiography (EKG) was done which demonstrated ST depression in leads I, aVL, V5 - V6, T wave inversion in lead III and $\mathrm{aVL}$; these findings were new compared to previous EKG. Laboratory results were as follows: hemoglobin 9.6 (reference value: $13.8-18 \mathrm{~g} / \mathrm{dL}$ ); platelet count $58 \times 10^{3} / \mu \mathrm{L}$ (reference value: $\left.150-450 \times 10^{3} / \mu \mathrm{L}\right)$; segmented neutrophils $80 \%(25$ $62 \%$ ); troponin $<0.03 \mathrm{ng} / \mathrm{mL}$ (reference value: $<0.03 \mathrm{ng} / \mathrm{mL}$ ); lactic acid $2.5 \mathrm{mmol} / \mathrm{L}$ (reference value: $0.5-2 \mathrm{mmol} / \mathrm{L}$ ); total bilirubin $5.4 \mathrm{mg} / \mathrm{dL}$ (reference value: 0.3 - $1 \mathrm{mg} / \mathrm{dL}$ ); direct bilirubin $0.9 \mathrm{mg} / \mathrm{dL}$ (reference value: 0 - $0.2 \mathrm{mg} / \mathrm{dL}$ ); reticulocyte count $3.8 \%$ (reference value: $0.7-1.7 \%$ ); lactate dehydrogenase (LDH) $478 \mathrm{U} / \mathrm{L}$ (reference value: 140 - $271 \mathrm{U} / \mathrm{L}$ ); haptoglobin $<30 \mathrm{mg} / \mathrm{dL}$ (reference value: $44-215 \mathrm{mg} / \mathrm{dL}$ ). His iron, vitamin B 12, thyroid stimulating hormone (TSH) and folic acid were within normal range. Ferritin level was not checked. His prior hemoglobin approximately 11 months to admission was $15.4 \mathrm{~g} / \mathrm{dL}$. Prior EKG did not show any gross ST segment or T-wave changes but did show q waves in lead II, III, V4, V5 and V6. Computed tomography (CT) abdomen and pelvis with IV contrast was done which was unremarkable except for splenomegaly $(16 \mathrm{~cm})$. Transthoracic echocardiography showed normal ejection fraction and moderate aortic stenosis. Blood smear was urgently done, and it indicated spherocytes without evidence of schistocytes. Patient was admitted with concern for possible AIHA and UA.

Cardiology consultant evaluated the patient and observed patient's presentation was consistent with UA with symptoms also being contributed by anemia. Hematology also evaluated the patient and determined that patient's presentation was likely secondary to ES due to presence of hemolysis and thrombocytopenia. They also observed other possibilities in- cluded paroxysmal nocturnal dyspnea despite AIHA being more likely due to smear findings. Direct antiglobulin test was obtained, and it came back positive for IgG confirming the diagnosis of AIHA. Due to concurrent likely immune-mediated thrombocytopenia, the diagnosis of ES was established. Patient was also considered to have concurrent UA which can be categorized as a thrombotic event despite ES's association with thrombocytopenia.

\section{Treatment}

Patient was started on prednisone $80 \mathrm{mg}$ daily. Patient continued to have anemia and after two doses, prednisone was switched to dexamethasone $40 \mathrm{mg}$ once daily for 4 days. Patient underwent cardiac stress tests once he clinically improved which indicated mild reversible inferior apical ischemia. Cardiac catheterization was pursued, and it revealed 95\% stenosis of proximal left circumflex artery requiring single drug eluding stent placement. Dual antiplatelet therapy was also initiated due to high risk of stent thrombosis despite ongoing intermittent transfusions.

Patient continued to have anemia (dropped from 9.6 to 7.6 $\mathrm{g} / \mathrm{dL}$ ) despite five intermittent blood transfusions, although his platelet counts improved $\left(58 \times 10^{3} / \mu \mathrm{L}\right.$ to $\left.88 \times 10^{3} / \mu \mathrm{L}\right)$. Patient remained hemodynamically stable. Later his hemoglobin dropped to $7.1 \mathrm{~g} / \mathrm{dL}$ and by this time he had received a total of eight packed red blood cell (PRBC) transfusions since admission. He was considered to be a candidate for rituximab, and he was started on $375 \mathrm{mg} / \mathrm{m}^{2}$ every week for total four doses. Patient's hepatitis B core antibody later was noted to be positive with a negative hepatitis surface antigen and positive hepatitis $\mathrm{B}$ surface antibody. There was a concern for risk of reactivation of hepatitis B with rituximab for which he was started on entecavir prophylactically. His hemoglobin dropped to $6.3 \mathrm{~g} /$ $\mathrm{dL} 2$ days after receiving the rituximab, which prompted the involvement of surgical consultants for possible splenectomy. However, subsequently after two more units of hemoglobin his hemoglobin stabilized, and splenectomy was deferred.

\section{Follow-up and outcomes}

Patient's hemoglobin stabilized after first dose of rituximab. IV steroids were transitioned to oral prednisone $90 \mathrm{mg}$ once daily. He was continued on dual antiplatelet therapy which he tolerated well, and thrombocytopenia continued to improve. Patient's chest pain resolved post cardiac catheterization. Once patient clinically improved, he was discharged with plans for slow tapering of steroids of prednisone $90 \mathrm{mg}$ and recommendations for outpatient cardiology and hematology follow-up. Plan was also to continue rituximab weekly for a total of four doses.

\section{Discussion}

"Primary Evans syndrome (ES) is defined by the concurrent 
or sequential occurrence of immune thrombocytopenia and autoimmune hemolytic anemia in the absence of an underlying etiology" [6]. Diagnosis of primary ES is established once other etiologies such as primary immunodeficiency, systemic lupus erythematosus and thrombotic thrombocytopenic purpura (TTP) are ruled out [5]. If patient is anemic, a smear should be done promptly which may show spherocytes, which may help in diagnosis as it is indicative of autoimmune hemolysis. The absence of schistocytes can be considered a clue to rule out TTP as was the case in our patient. Coombs's test can also be done which can further assist in establishing diagnosis. Other tests indicating hemolytic anemia include LDH, haptoglobin, reticulocyte count and bilirubin levels. Often, ES can have relapsing and remitting course requiring recurrent interventions. Due to the rarity of syndrome itself, no randomized studies focusing on treatment are available till date and therapy is mainly tailored towards addressing AIHA and ITP [5]. "Corticosteroids represent the cornerstone therapy, used at a daily dose of $1 \mathrm{mg} / \mathrm{kg}$ of prednisone" [7]. Patients who do not respond to steroids may require rituximab, IVIG or even splenectomy.

ACS can rarely occur with ES and is associated with high morbidity and mortality, necessitating early reperfusion [6]. As this is a thrombotic process, it can be difficult to diagnose in the setting of ES which commonly presents with thrombocytopenia. The pathophysiology of the thrombotic events in ES is not clearly understood. One hypothesis revolves around association of AIHA with thrombotic events since ES manifests with AIHA as well. AIHA has been associated with thrombotic events and according to literature $15-33 \%$ adults with warm AIHA may have VTEs [8]. However, considering ES patient also have thrombocytopenia, the mechanism of thrombosis in such patients is not clearly understood. There are a limited number of cases reports or case series emphasizing on the occurrence of thrombotic events in ES.

In our patient, normal troponin levels further complicated the situation making it harder to determine if underlying cardiac ischemia was present. However, there was high suspicion due to the character of his chest pain and risk factors. A cardiac stress test was pursued, and it was positive for ischemia. Multivessel disease was later confirmed through cardiac catheterization leading to stent placement which also presented a new challenge in the form of necessity of dual antiplatelets and risk of bleeding due to anemia as well as thrombocytopenia. Although platelets contribute to occurrence of ACS, thrombocytopenia does not result in reduction of this problem [9]. Hence, in our patient dual antiplatelet therapy was continued.

As the patient had risk factors for coronary artery disease these risk factors can be the primary reasons for UA. Considering recent onset of chest pain and concurrent presence of ES, it was considered that UA may have occurred due to thrombotic process secondary to ES, although it is difficult to establish this with certainty.

Patient did not initially respond to oral steroids which complicated the hospital course further. Prednisone was transitioned to IV dexamethasone, although there is limited evidence of any significant benefit to patients who do not respond to steroids. Eventually he was started on rituximab infusion which resulted in stabilization of his hemoglobin levels. He only required two more units of PRBC transfusions after rituximab infusion and thereafter his hemoglobin was noted to uptrend. Prior to initiation of rituximab, patient had required eight units of PRBC transfusions. This highlights the importance of transitioning from steroids of immunotherapy in non-responsive cases. Rituximab which has been approved for lymphomas has also been used to treat ITP and AIHA such as ES [10].

An interesting factor of this case was positive results for core hepatitis B antibody. There have been reported case reports of ES secondary to hepatitis B. In addition, the US Food and Drug Administration issued a new boxed warning of hepatitis B virus (HBV) reactivation after 109 cases of fatal reactivation of hepatitis $B$ in patients who were receiving rituximab [11]. In our patient considering his hepatitis B surface antibody was positive he was considered not to have active hepatitis B infection. However, it was deduced that there may be residual $\mathrm{HBV}$ in the liver with potential for reactivation with rituximab. Hence, he was prophylactically given entecavir which is an inhibitor of replication of HBV [12].

\section{Learning points}

ES is a rare disorder which can present with anemia, thrombocytopenia, and neutropenia. Although it is usually associated with thrombocytopenia it can sometimes lead to thrombotic processes. ACS can rarely present in the setting of ES which can be challenging to manage considering the treatment may require stent placement and dual antiplatelet therapy in the setting of anemia and thrombocytopenia. The treatment of ES involves steroids which can be followed by rituximab, IVIG or splenectomy for non-responsive cases. If a thrombotic event such as ACS occurs due to ES, patients may require reperfusion and dual antiplatelet therapy. Early intervention and management can prevent mortality and morbidity.

\section{Acknowledgments}

None to declare.

\section{Financial Disclosure}

No funding was obtained in preparation of this manuscript.

\section{Conflict of Interest}

Authors declare no conflict of interest.

\section{Informed Consent}

Not applicable. 


\section{Author Contributions}

All authors have contributed to the manuscript preparation. Each author has reviewed the final version of the manuscript and approves it for publication.

\section{Data Availability}

The authors declare that data supporting the findings of this study are available within the article.

\section{Abbreviations}

ES: Evans syndrome; AIHA: autoimmune hemolytic anemia; UA: unstable angina; ITP: immune thrombocytopenic purpura; IgG: immunoglobulin G; IVIG: intravenous immunoglobulin; RBC: red blood cells; EKG: echocardiography; CT: computed tomography

\section{References}

1. Couri FS, Kandula M. A case of Evans syndrome with acute hemolysis and hemoglobin cast nephropathy. Am J Case Rep. 2020;21:e920760.

2. Al Hazmi A, Winters ME. Evans syndrome. Clin Pract Cases Emerg Med. 2019;3(2):128-131.

3. Michel M, Chanet V, Dechartres A, Morin AS, Piette JC, Cirasino L, Emilia G, et al. The spectrum of Evans syndrome in adults: new insight into the disease based on the analysis of 68 cases. Blood. 2009;114(15):3167-3172.

4. Shaikh H, Mewawalla P. Evans syndrome. [Updated Jun 26, 2021]. In: StatPearls [Internet]. Treasure Island (FL): StatPearls Publishing; Jan 2021. Available from: https:// www.ncbi.nlm.nih.gov/books/NBK519015.

5. Kizilirmak F, Demir GG, Cakal B, Bekoz HS, Olgun FE. Evans syndrome with non-ST segment elevation myocardial infarction complicated by hemopericardium. Indian Heart J. 2016;68(Suppl 2):S280-S283.

6. Mantadakis E, Farmaki E. Natural history, pathogenesis, and treatment of Evans syndrome in children. J Pediatr Hematol Oncol. 2017;39(6):413-419.

7. Audia S, Grienay N, Mounier M, Michel M, Bonnotte B. Evans' syndrome: from diagnosis to treatment. J Clin Med. 2020;9(12):3851.

8. Audia S, Bach B, Samson M, Lakomy D, Bour JB, Burlet B, Guy J, et al. Venous thromboembolic events during warm autoimmune hemolytic anemia. PLoS One. 2018;13(11):e0207218.

9. Susilovic Grabovac Z, Bakovic D, Lozo M, Pintaric I, Dujic Z. Early Changes in Platelet Size and Number in Patients with Acute Coronary Syndrome. Int J Angiol. 2017;26(4):249-252.

10. Park CY, Chung CH. A patient with mixed type Evans syndrome: efficacy of rituximab treatment. J Korean Med Sci. 2006;21(6):1115-1116.

11. Martin ST, Cardwell SM, Nailor MD, Gabardi S. Hepatitis $\mathrm{B}$ reactivation and rituximab: a new boxed warning and considerations for solid organ transplantation. Am J Transplant. 2014;14(4):788-796.

12. Dimou E, Papadimitropoulos V, Hadziyannis SJ. The role of entecavir in the treatment of chronic hepatitis B. Ther Clin Risk Manag. 2007;3(6):1077-1086. 